\title{
Silver nanoplates on graphite substrates for ultrasensitive and label free Surface-Enhanced Raman Scattering (SERS) based detection of organophosphorous nerve agents in gas phase
}

\author{
M. Lafuente ${ }^{1}$, D. Sanz ${ }^{1}$, M. Urbiztondo ${ }^{2}$ R. Mallada ${ }^{1,3}$, M.P. Pina ${ }^{1,3}$, J. Santamaría ${ }^{1,3}$ \\ ${ }^{1}$ Nanoscience Institute of Aragon, University of Zaragoza, Department of Chemical \& Environmental \\ Engineering, Edif. I+D+i, Campus Rio Ebro, C/Mariano Esquillor, s/n, 50018 Zaragoza, Spain. \\ ${ }^{2}$ Centro Universitario de la Defensa de Zaragoza, 50090 Zaragoza, Spain \\ ${ }^{3}$ Networking Research Center on Bioengineering, Biomaterials and Nanomedicine, CIBER-BBN, \\ 28029 Madrid, Spain. \\ mapina@unizar.es
}

\begin{abstract}
A surface-enhanced Raman scattering (SERS)-based sensor has been developed for the label-free real-time gas phase detection of dimethyl methylphosphonate (DMMP); a surrogate molecule of the Gseries nerve agents which are of particular concern due to its extreme toxicity, persistence and previous deployment. In particular, SERS substrates based on high density Ag nanoplates (LSPR at $700 \mathrm{~nm}$ ) monolayers on graphite have been straightforwardly attained by electrostatically-driven adsorption technique. Unlike Au based counterparts, SERS measurements suggest the chemisorption of DMMP molecules to the Ag nanoplate surface, eventually leading to photoinduced decomposition products. These SERS substrates have demonstrated the capability for the reliable identification of DMMP in gas phase at $2.5 \mathrm{ppmV}$, with the advantages of simple operation and low cost.
\end{abstract}

Key words: SERS, neurotoxic agents identification, gas phase, real time detection, sub-ppmV

\begin{abstract}
Introduction
The G-series nerve agents, i.e. Tabun (GA), Sarin (GB), Soman (GD), are widely recognized as one of the most toxic group of chemical warfare agents (CWA) due to the presence of organophosphorus esters causing systemic effects predominantly on the central nervous system. Nowadays, the competing technologies for the rapid detection of chemical agents in gas phase face severe limitations in terms of specificity, portability, cost and simplicity.
\end{abstract}

SERS is one of the leading techniques for labelfree ultrasensitive vibrational fingerprinting of a variety of molecular compounds [1]. In the field of explosives and chemical threat detection [23], SERS has been identified as key technology thanks to distinctive features such as: ultrahigh sensitivity, detection from a wide variety of matrices and quantification of multiple species in a single measurement, allowing real time detection in the field.

SERS detection at trace concentration levels in gas phase is hampered by the fact that only a few molecules of interest are localized in very close vicinity (typically less than $10 \mathrm{~nm}$ ) of the metal when localized surface plasmon nodes are excited. Many strategies address this problem, including the use of partition layers with low affinity and rapidly reversible binding sites [4-5] or the formation of stable complexes on the plasmonic surface [6]. In this work, real time SERS identification of DMMP has been demonstrated with Ag nanotriangle monolayers on graphite at $2.5 \mathrm{ppmV}$ concentration. Their sensing performance has been assessed by comparison with $\mathrm{Au}$ based SERS substrates, already developed in our group [4].

\section{Preparation and Characterization of SERS substrates}

The citrate capped Ag nanotriangles were synthesized according to published recipe [8]. The average edge length of $\mathrm{Ag}$ nanoplates is $38 \mathrm{~nm}$ (see Fig. 1) with the LSPR centred at $700 \mathrm{~nm}$. The $\mathrm{Ag}$ assembly process was performed on graphite substrates previously coated with PDDA according to our previous works [4-5]. Graphite provides both 
fluorescence quenching and surface area for nanoparticle dispersion.

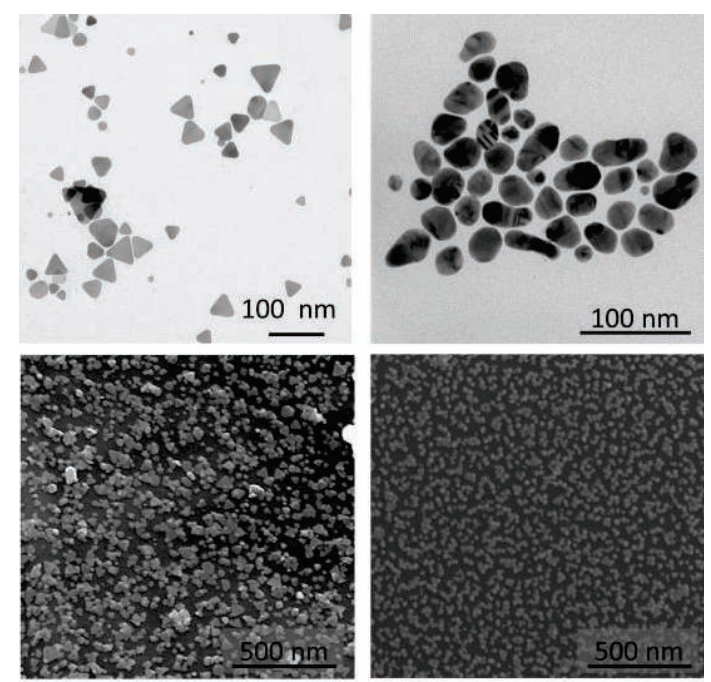

Fig. 1. TEM images of $\mathrm{Ag}$ (left) and $\mathrm{Au}$ (right) nanostructures (upper part), and SEM analysis of their assembled monolayers on graphite and $\mathrm{SiO}_{2} / \mathrm{Si}$ respectively (botton part).

Raman experiments were conducted using Alpha 300 Raman spectrometer (WITec) with excitation wavelength of $785 \mathrm{~nm}$ and optical lens $\times 50$. R6G was chosen as probe molecule and the calculated EF and SERS gain (refer to [5] for details on calculations) are presented in Tab. 1. The EF and SERS gain values from $\mathrm{Au}$ assemblies were circa one order of magnitude weaker than that from $\mathrm{Ag}$, in agreement with the electromagnetic theory. However, the superior SERS performance of $\mathrm{Ag}$ is hampered by its poor stability, i.e. EF and SERS gain decays almost one order of magnitude on samples exposed to room conditions (aged) for 2 months. Our efforts are devoted to ondemand assembly of active SERS substrates directly prior to experiment to minimize potential ambient degradation of the Ag surfaces.

Tab. 1: Enhancement factor and SERS gain for the $A g$ and $A u$ based substrates studied in this work.

\begin{tabular}{|c|c|c|c|}
\cline { 2 - 4 } \multicolumn{1}{c|}{} & $\begin{array}{c}\text { Ag_Graphite } \\
\text { (fresh) }\end{array}$ & $\begin{array}{c}\text { Ag_Graphite } \\
\text { (aged) }\end{array}$ & Au_SiO $_{2} / \mathrm{Si}$ \\
\hline EF & $5.31 \times 10^{5}$ & $7.79 \times 10^{4}$ & $7.21 \times 10^{4}$ \\
\hline Gain & $1.33 \times 10^{8}$ & $2.53 \times 10^{7}$ & $1.80 \times 10^{7}$ \\
\hline
\end{tabular}

\section{SERS Detection in Gas Phase of DMMP}

A nitrogen stream containing $2.5 \mathrm{ppmV}$ of DMMP was fed to the home-made exposure cell where the SERS substrate was mounted [45]. The characteristic Raman signature of DMMP, with main bands at 515, 710 and 814 $\mathrm{cm}^{-1}$, are clearly developed upon $15 \mathrm{~min}$ exposure on $\mathrm{Ag}$ substrates (see Fig. 2). The limit of detection of DMMP could be down to $725 \mathrm{ppb}$. Opposed to Au substrates, the Raman bands of citrate species are attenuated, and the photo degradation of the target analyte is diminished thanks to graphite. Analyzing the time-dependent evolution of SERS spectra, chemisorption of DMMP molecules and subsequent decomposition reaction on $\mathrm{Ag}$ surface is postulated. Further efforts are focused on DFT calculations to get insight the formation of Ag-DMMP complexes.

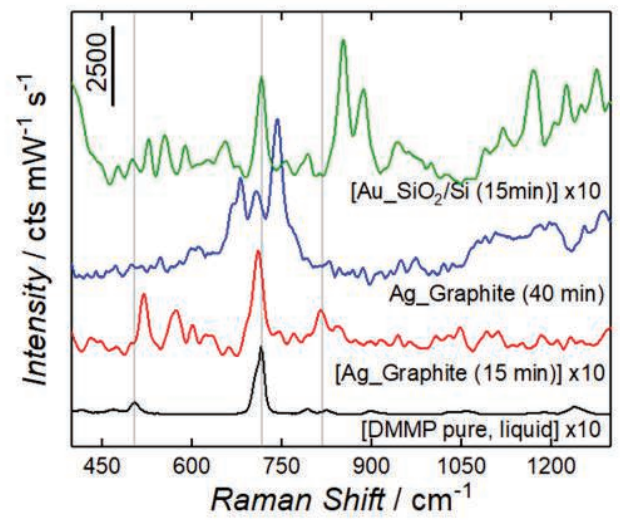

Fig. 2. SERS spectra of high-density $\mathrm{Au}$ and $\mathrm{Ag}$ monolayers during exposure to 2.5 ppmV DMMP.

\section{References}

[1] B. Sharma, et al., SERS: Materials, applications, and the future, Materials Today 15, 16-25 (2012); doi: 10.1016/S1369-7021(12)70017-2

[2] R. S. Golightly, et al., Surface-Enhanced Raman Spectroscopy and Homeland Security: A Perfect Match? ACS Nano 3, 2859-2869 (2009); doi: 10.1021/nn9013593

[3] A. Hakonen, et al., Explosive and chemical threat detection by surface-enhanced Raman scattering: a review, Anal. Chim. Acta 893, 1-13 (2015); doi: 10.1016/j.aca.2015.04.010

[4] M. Lafuente, et al., 3D Fractals as SERS Active Platforms: Preparation and Evaluation for Gas Phase Detection of GNerve Agents, Micromachines 9 (60), 1-14 (2018); doi:10.3390/mi9020060

[5] M. Lafuente, et al., Highly sensitive SERS quantification of organophosphorous chemical warfare agents: a major step towards the real time sensing in the gas phase, Sens. Actuator B-Chem. submitted

[6] R. K. Lauridsen, et al., Towards quantitative SERS detection of hydrogen cyanide at ppb level for human breath analysis, Sensing and Bio-Sensing Research 5, 84-89 (2015); doi: 10.1016/j.sbsr.2015.07.002

[7] J. Frank, et al., Synthesis of Silver Nanoprisms: Exploring Plasmonic Nanoparticles, J. Chem. Educ., 87 (10), 1098-1101 (2010); doi: 10.1021/ed100166g 